Cahiers d'études africaines

187-188|2007

Les femmes, le droit et la justice

\title{
Procès au féminin et changements de société
}

\section{Catherine Coquery-Vidrovitch}

\section{OpenEdition}

Journals

Édition électronique

URL : http://journals.openedition.org/etudesafricaines/7732

DOI : 10.4000/etudesafricaines.7732

ISSN : 1777-5353

\section{Éditeur}

Éditions de l'EHESS

\section{Édition imprimée}

Date de publication : 15 décembre 2007

Pagination : 449-460

ISBN : 978-2-7132-2140-8

ISSN : 0008-0055

\section{Référence électronique}

Catherine Coquery-Vidrovitch, "Procès au féminin et changements de société », Cahiers d'études africaines [En ligne], 187-188 | 2007, mis en ligne le 03 janvier 2008, consulté le 24 septembre 2020. URL : http://journals.openedition.org/etudesafricaines/7732 ; DOI : https://doi.org/10.4000/ etudesafricaines.7732

Ce document a été généré automatiquement le 24 septembre 2020.

(c) Cahiers d'Études africaines 


\title{
Procès au féminin et changements de société
}

\author{
Catherine Coquery-Vidrovitch
}

1 Ce numéro consacré aux rapports entre les femmes africaines et le droit, vus à travers les archives de justice, voudrait inciter à faire avancer la recherche au-delà de ses propos actuels. L'idée de départ vient d'Afrique, et plus précisément d'une conférence internationale organisée par le directeur des Archives nationale du Sénégal d'alors, Saliou Mbaye, qui entendait attirer l'attention sur des sources jusqu'alors très négligées par les chercheurs : les Archives juridiques. D'où une rencontre sur le thème « Archives judiciaires, sciences sociales et démocratie» tenue à Dakar en décembre 2003. La découverte fut passionnante, et on y parla un peu des femmes. Mais encore bien peu. De là, vint l'idée de centrer un Cahier sur cette question encore plus négligée que le reste. L'appel d'offres a dépassé nos espérances.

2 Les acquis sont rassemblés dans le premier article qui s'efforce de faire le point de la littérature existante sur les rapports entre les femmes et le droit en longue durée, depuis la veille de la colonisation jusqu'à l'époque contemporaine. Or, si les connaissances ainsi rassemblées sont considérables, elles s'inscrivent néanmoins, dans l'ensemble, dans le schéma classique de l'analyse des catégories occidentales; le droit est réparti en sections bien distinctes mises en valeur par les codes et les recueils de jurisprudence : public/privé, économique/social, droit foncier, droit des affaires, droit commun/affaires politiques, voire droit religieux (dans les procès en sorcellerie). Or, les études de cas démontrent, au contraire, à quel point ces différents éléments peuvent être liés dans la même affaire. L'importance même du divorce le révèle : le concubinage, l'«adultère " (notion fondamentalement occidentale) ou le divorce n'étaient pas, comme en Occident, des affaires purement privées, «domestiques ». Les articles de ce recueil insistent notamment sur la place tenue par la compensation matrimoniale (dite « dot » pour aller vite) dans les constructions sociales, par le rôle primordial d'« outil de production » tenu par les épouses, par l'importance du lévirat dans les conflits fonciers qui en découlent, en particulier dans les affaires de veuvage et 
d'héritage. Bref, toutes les structures de la société furent menacées ou ébranlées par la prise progressive d'émancipation individuelle revendiquée par les femmes.

Parler de divorce, ce n'est donc pas seulement focaliser sur une étude des stratégies individuelles au niveau du droit de la famille. Naguère, la rupture du mariage avait peu à voir avec une simple affaire de sentiment ou d'appartenance familiale. Aujourd'hui, c'est l'ensemble du corps social qui a été remis en jeu par un autre mode de circulation des femmes : l'épouse qui refuse d'être achetée par une dot bouleverse l'organisation $\mathrm{du}$ travail rural; celle qui refuse l'anneau conjugal pour devenir chef de famille indépendante et chef d'entreprise à son compte bouleverse l'économie urbaine. Il faut se détacher de la vision coloniale bien pensante de l'ère bourgeoise victorienne, distillée par la lecture des archives, pour repenser le processus: les recherches ont surtout insisté sur les procédures de divorce en tant que remise en cause individuelle du mode de vie conjugal, en y voyant le signe évident d'une évolution des mœurs et de la morale. On peut poser la question d'une autre manière: le divorce, institution désormais fréquente et même souvent largement majoritaire dans de nombreuses sociétés africaines, pas nécessairement urbaines, n'est-il pas en passe de devenir l'un des pivots fondamentaux de la remise en question de l'organisation socio-économique contemporaine? Après tout, Claude Meillassoux l'avait déjà pressenti en 1960, et à nouveau théorisé en 1976, la circulation des femmes fut autrefois, par le truchement de la dot, une des clés de l'histoire africaine ancienne ${ }^{1}$. Le divorce, acte social, économique, voire politique qui révèle l'émancipation féminine, n'est-il pas aujourd'hui, à nouveau, mais de façon neuve, "moderne", originale, à la fois un catalyseur et un révélateur de la transformation de la société dans son ensemble, par la prise en main "genrée» de la dynamique sociale? C'est un peu ce que certains analystes d'aujourd'hui prédisent, en affirmant le rôle prédominant que devrait jouer dans l'avenir de l'Afrique le genre féminin...

Dans le premier article, Catherine Coquery-Vidrovitch rassemble le savoir existant sur les rapports entre les femmes et le droit en longue durée. Les recherches en langue anglaise ont démarré plus tôt et ont donc une longueur d'avance sur les travaux de langue française dont on trouve dans ce numéro les principales promesses. On y constate aussi que les chercheurs anglophones se sont récemment attaqués au domaine $\mathrm{du}$ droit francophone, dont la connaissance devient donc analogue au domaine anglophone. A travers les sources juridiques, on perçoit plusieurs niveaux de droit parfois entrecroisés : à partir d'un héritage ancien qui a lui-même évolué au fil des siècles précoloniaux, le colonisateur a, avec le concours des chefs et autres notables, en partie " inventé » un droit dit coutumier qui fut et demeure (surtout en pays d'héritage britannique) imbriqué dans le droit «moderne » d'inspiration occidentale. Les affaires traitées au tribunal permettent d'éclairer l'évolution de trois thèmes majeurs dans lesquels interviennent, de façon spécifique, les femmes: celui de la sorcellerie (peu abordé ici par ailleurs); celui, très privilégié, des rapports de conjugalité et de la libéralisation progressive du divorce à l'initiative des femmes; enfin celui du droit de propriété différencié entre hommes et femmes, qui est parfois mais pas toujours lié au droit de la famille, si ce n'est dans les affaires de compensation matrimoniale ou d'héritage.

5 Odile Goerg, à propos de l'exercice de la justice en Guinée, centre son analyse sur l'histoire genrée du droit colonial à la française. Elle série l'intérêt et les limites des sources disponibles : car, bien entendu, toute la justice ne passe pas au tribunal, et donc 
tout n'en a pas été enregistré par les instances coloniales. Une partie importante des litiges a été réglée de façon autonome sans que les observateurs et plus précisément les administrateurs coloniaux en aient eu connaissance. À partir de là, qu'est-ce qui détermine la spécificité des cas et du recours en justice entre les sexes? Comment apprécier la différence évidente des délits ciblés selon le genre de l'accusé ou du plaignant : ce qui est jugé comme déviant, est-ce une réalité sociale, ou bien cela relèvet-il du choix et des préjugés du colonisateur? Il y a sûrement un peu des deux; une chose est sûre, même si les archives sont rarement sexuées: sur l'inventaire très soigneusement dressé, de tout ce qu'Odile Goerg a pu répertorier entre 1925 et 1939 dans les archives juridiques, par classe d'âge, par sexe, par type de délit, par lieu, en forte majorité les femmes apparaissent lors de questions de droit privé, alors que les hommes ont à gérer des délits classiques de société : vols, voire assassinat, ou détérioration de biens. Les femmes, elles, apparaissent dès que leur mariage se passe mal: problèmes de dot, d'adultère, de divorce surtout dominent largement. Cela permet d'axer ensuite le développement sur l'imbroglio des affaires matrimoniales, même si celles-ci apparaissent nettement moins abordées que dans d'autres colonies, car le nombre de femmes concernées constitue une toute petite minorité face au total des affaires traitées. Le nombre de divorces demandés par les épouses progresse lentement. Cela dénote le retard des mutations sociales face aux changements économiques et politiques. Peut-on avancer une hypothèse ? Cette absence prolongée d'émancipation des femmes guinéennes aide sans doute à rendre compte de la popularité " genrée » à venir de Sekou Touré, qui fera de la politique féminine l'un de ses chevaux de bataille ${ }^{2}$.

6 Lily Mafela nous entraîne à l'autre bout de l'Afrique pour l'évolution du droit concernant les femmes du Botswana dont l'histoire coloniale a commencé beaucoup plus tôt (1840-1980). Elle en dresse surtout le cadre sociétal porteur. Ce qui rapproche les femmes de Guinée de celles du Botswana, c'est la situation de subordination totale des femmes à l'autorité patriarcale masculine, depuis l'enfance de la fillette jusqu'au quasi-esclavage de la femme mariée dans la société ancienne, dont les usages ont pu se prolonger fort tard. Bref, le dicton enraciné chez les Tswana selon lequel « la femme est l'enfant de l'homme » n'a guère été amélioré par l'idéologie missionnaire de soumission chrétienne de l'épouse au chef de famille. L'auteure montre néanmoins le lien entre deux puissants catalyseurs de changement social dans les rapports genrés : la migration (aussi bien des femmes que des hommes) et l'éducation, même empreinte de l'esprit victorien de la femme-mère au foyer. Du côté africain comme du côté colonial, la réaction des autorités fut de s'opposer par tous les moyens possibles - donc prioritairement par le recours à la coutume et à l'appareil juridique colonial - à la migration du travail des femmes, car celle-ci signifiait un risque réel d'émancipation. Paradoxalement, ces contraintes de stabilité favorisèrent parfois une scolarisation plus poussée des filles que des garçons (ceux-ci requis par les rotations du travail minier). Mais, compte tenu des énormes réticences héritées de la colonisation, ce n'est que depuis peu que certaines femmes éduquées peuvent en recueillir le fruit en revendiquant leur autonomie au sein du foyer conjugal.

7 Marc Le Pape, un peu comme Odile Goerg pour la Guinée, fait pour Abidjan (Côted'Ivoire) l'inventaire des conflits juridiques impliquant des femmes entre les deux guerres (1923-1939). Une fois encore, ce sont les affaires privées qui priment. Il relève, de la même façon, le faible taux d'affaires féminines tournant autour d'autres délits. Il s'agit le plus souvent de divorce, avec des arguments analogues concernant la dot ou 
l'escroquerie au mariage. Mais bien d'autres motifs de discordes apparaissent: notamment la violence de l'homme qui bat fréquemment ses femmes; comme nous sommes en ville et dans des quartiers populaires, les femmes manifestent aussi leur indépendance, les rixes entre femmes elles-mêmes ne sont pas rares, pour les motifs les plus divers: jalousies amoureuses, mais aussi vols de pagnes, commerce illicite, querelles de voisinage. La justice s'avère tâtonnante, reconnaissant de facto qu'il n'y a pas de "vraie " coutume, sinon celle que le tribunal est amené à stipuler lui-même. Bref le droit, témoin de mœurs changeantes, révèle un ensemble d'usages en incessant devenir.

Un certain nombre des articles suivants privilégie l'étude de cas précis; leur point commun est d'être souvent (mais pas seulement) centrés sur le divorce et son évolution vue à travers les jugements collectés : ceux-ci sont nombreux pour chacune des études, le plus souvent supérieurs à une ou plusieurs centaines, ce qui permet de tirer des conclusions convaincantes. L'une d'entre elles est que le «droit au divorce » apparaît incontestablement comme une conquête féminine.

9 Marie Rodet montre, à propos du Soudan français (Mali), entre les deux guerres, à l'occasion de deux cas similaires situés dans la même ville de Kayes à vingt ans d'intervalle (1918 et 1939), à quel point l'administration coloniale avait choisi, dans cette région sahélienne éloignée de l'Afrique «utile» et urbaine, de privilégier la coutume. Elle s'appuya sur le conservatisme masculin des chefs, pour lutter contre la mobilité des femmes et leur aspiration au divorce. Néanmoins, les femmes avaient compris dès le début de l'occupation française que la fluctuation des positions leur offrait un jeu de négociation possible ; dès le premier cas, l'énergie de la plaignante et la variété de ses interventions témoignent de sa connaissance des changements en cours. Néanmoins, il était encore trop tôt et elle fut contrainte de retourner chez son mari. En revanche, le conflit quasi analogue qui éclata en fin de période et qui donna à peu près autant de tracas à la justice et à l'administration coloniale saisie de façon hiérarchique successive jusqu'au plus haut niveau, permit en définitive à la femme d'avoir gain de cause. À l'occasion de ces affaires révélant les contradictions inhérentes entre droit coutumier et liberté individuelle, où dans l'un et l'autre cas (et ceux-ci ne furent pas uniques) l'épouse alla au bout des recours possibles, démonstration est faite que les temps avaient quand même changé : les femmes en furent en ce domaine du droit privé le moteur essentiel.

10 Avec Emily Burrill, nous percevons néanmoins à Sikasso la difficulté du combat pour les femmes dans les années 1930. C'est que relativement nombreuses sont celles qui demandent le divorce pour un motif peu abordé jusqu'à présent: la violence domestique et le viol. Dans cette région déjà traumatisée par les mutations sociales, la crise économique et le départ de nombreux travailleurs migrants, les coutumes patriarcales ont paradoxalement tendance à se durcir; le mari, quand il revient, dispose de tous les droits sur ses épouses. Pour peu qu'il soupçonne un adultère ou que la femme ait refait sa vie en son absence, qu'il ne soit pas satisfait de son travail ou qu'il cherche lui-même à s'en débarrasser, il la bat allègrement. Pire, les homicides ne sont pas rares, les cas de viols non plus. L'auteure s'appuie sur une série de dépositions de femmes soigneusement analysées qu'elle a dépouillées dans les archives locales. Les victimes se défendent âprement, mais ont bien du mal à faire reconnaitre le bien-fondé de leur plainte. Elles ne reçoivent pas toujours, loin de là, le soutien de la loi coloniale ni, plus surprenant, celui des autres femmes, dans la mesure où l'une et les autres 
s'appuient sur les mêmes arguments pour contribuer à les débouter : les convenances coutumières plus ou moins reconstituées et rigidifiées dont le recours permet de rejeter leurs allégations et minimiser leurs preuves.

11 Même si son importance a été exagérée par les anthropologues, le viol a toujours existé ; peu d'études ont été faites sinon le travail de Brett Shadle sur les Gusii du $\mathrm{Kenya}^{3}$. En ce cas, il faut néanmoins le différencier d'une coutume enracinée qui était celle de l'enlèvement des filles à marier, quasi seul moyen pour un jeune homme peu fortuné de convaincre un père de lui donner sa fille pour épouse. Cela n'a pas empêché le viol stricto sensu d'être aussi pratiqué. Mais il a été très peu étudié par les chercheurs, sinon dans le cas du viol d'une femme blanche par un noir, ce qui était sous la colonisation un crime puni de mort. Le viol d'une femme africaine n'était guère pris en compte par la justice coloniale, car les colonisateurs (et les missionnaires) ne comprenaient pas cette culture où les relations sexuelles étaient assez libres entre garçons et filles dès la puberté et avant le mariage. Ils refusaient donc de reconnaître qu'il y ait une différence que la fille ait été ou non forcée. Paradoxalement, c'est le droit coutumier qui était, de façon probablement assez ancienne, plus ouvert que le droit colonial ; les tribunaux coutumiers locaux se montraient attentifs : puisque les relations sexuelles pré-maritales étaient autorisées, si la fille se plaignait c'est qu'elle avait ses raisons et elle était écoutée. Mais le viol n'est devenu un cas de justice sévèrement puni que lorsque les femmes se mirent à porter plainte elles-mêmes, ce qui devient évident après la Deuxième Guerre mondiale.

12 Awa Yade s'attache à l'évolution des stratégies familiales au Sénégal. Comme dans les autres milieux urbains, elles ont beaucoup évolué. Elle conteste les idées reçues concernant l'idée que la colonisation aurait déséquilibré les rapports de genre en défaveur des femmes, en contribuant à leur perte de pouvoir et "d'autonomie sociale » au sein de la structure familiale. Elle part au contraire du constat que le mariage en Afrique a connu, dans le contexte matérialiste contemporain, des transformations analogues à celles des autres régions du monde. Naguère, le mariage préférentiel consistait à choisir pour son fils ou sa fille le mari ou l'épouse idéal. Ces unions, qui étaient autant d'alliances croisées, visaient à consolider les liens du groupe. Elles sont aujourd'hui considérées comme des mariages forcés dans la mesure où l'accord des époux n'était pas requis. La plupart des femmes rencontrées reconnaissent ne pas avoir vécu en leur temps ces unions comme telles, puisqu'il n'y avait pas d'autre alternative que de continuer sur les traces de la mère. Quant à l'idéologie coloniale française, elle jugeait la situation contraire aux principes des droits humains, surtout pour la femme. Dès 1903, un décret sur la justice en AOF a exigé, en principe, pour la femme, le droit de consentement au mariage, le droit à la propriété de la dot (qui existe dans le droit musulman), et le droit au divorce.

13 Le divorce ne fut pas une "invention » coloniale. Il était fréquent dans les milieux coutumiers, mais les causes admises par la coutume et l'islam tournaient exclusivement autour de la répudiation ou de la disparition de l'un des conjoints. À la faveur des bouleversements économiques et sociaux intervenus à partir de 1900, une pléthore d'autres motifs est venue se greffer sur celles-ci. Parallèlement, la monétarisation de l'économie a déteint sur les rapports sociaux, conduisant certains parents à se lancer dans « une vente aux enchères ». Les jeunes filles sont devenues pour leurs pères des sources de revenus, situation qui s'est aggravée avec la crise économique des années 1930. Pour mettre fin à ces abus, ce sont les femmes qui ont été les actrices principales 
de la multiplication du phénomène de divorce : sur plus d'une centaine de jugements recensés par l'auteure, l'initiative vient pratiquement toujours de l'épouse, pour des raisons diverses souvent relatives au paiement ou au remboursement de la dot (compensation matrimoniale). L'article analyse les causes majeures de divorce, de l'abandon du domicile conjugal à l'adultère, et l'évolution des mœurs qui en est résultée.

Rachel Jean-Baptiste analyse le même type de problèmes sur le cas du Gabon dans la période suivante, de 1939 à 1959. L'étude allie l'exhaustivité que permet l'analyse de plusieurs centaines de cas à l'exemplarité de certains exemples bien choisis. La confrontation est intéressante, car on passe d'un système quasi exclusivement musulman à un système chrétien, mais dans les deux cas greffé sur un solide substrat coutumier. Or on constate, malgré la différence d'époque, de mœurs et de religion, l'existence de traits communs, dans un contexte similaire de mutation économique, de dislocation sociale et de migration urbaine. Aussi bien au tribunal coutumier que devant la justice coloniale, les maris, les femmes et les partenaires hors mariage cherchent à définir à leur avantage comment peuvent se décliner le mariage, les droits et les devoirs réciproques du mari et de la femme, et les conditions exigées pour rompre le lien qui les lie. Comme ailleurs, la quasi-totalité des procès tourne autour de la question du paiement ou de la récupération de la dot, de l'enlèvement ou du départ de la femme, de l'adultère et du divorce. Beaucoup de ces affaires démontrent la façon dont les familles cherchent à tirer parti de la rentabilité de leurs filles. L'ambiguïté des réponses données aussi bien par le droit coutumier que par la loi coloniale ont offert aux individus une multitude de possibilités d'esquive ou de solution où les femmes ne sont pas les dernières à tirer leur épingle du jeu. Cela donne l'occasion d'une étude fouillée, d'une part du fonctionnement des institutions judiciaires et, d'autre part, des préoccupations contrastées des hommes et des femmes: comme au Sénégal, les Gabonais se montrent attachés à la stabilité du mariage - alliés en ceci aux administrateurs et aux missionnaires -, tandis que ce sont les Gabonaises qui aspirent au divorce et inventent des stratégies de remariage plus conformes à leurs goûts. L'étude souligne le rôle de la circulation de la dot dans le poids de la coutume du lévirat.

Dans un article riche de nombreuses références utiles pour le lecteur français, et en démarrant avec le cas de Makubutu au Lesotho dès 1874, Pule Phoopholo permet de nuancer en longue durée la périodisation de l'efficacité de l'action féminine d'émancipation conjugale. Il n'y eut pas deux phases d'évolution, mais au moins trois : dès l'arrivée des missionnaires, en l'occurrence en 1830, puis au début de la colonisation stricto sensu à la fin $\mathrm{du} \mathrm{XIX}^{\mathrm{e}}$ siècle, les femmes, très contraintes par la coutume, furent rapides à chercher à y échapper, et habiles à utiliser l'entregent des missionnaires ou des premiers administrateurs pour parvenir à leurs fins. L'article expose dans le détail les usages matrimoniaux avant cette intervention, ce qui permet de bien comprendre le processus. Au démarrage, les missionnaires, scandalisés par des mœurs qu'ils ne comprenaient pas, par la polygamie et par le fait qu'une veuve se voyait retirer ses enfants si elle n'acceptait pas le lévirat, considérèrent que les épouses étaient traitées comme des marchandises, des "beasts of burden " ${ }^{4}$, et assimilèrent le paiement et la circulation des dots au commerce des femmes - ce qui n'était pas faux. Voulant faire passer le mariage du statut de contrat de travail entre deux familles à celui de sacrement chrétien, ils écoutèrent d'une oreille attentive les complaintes des femmes. Celles-ci, dans la quasi-totalité des dépositions, se montrent offensives et 
décidées. Elles voulaient échapper à des maris abusifs qui les maltraitaient, les battaient, ou les faisaient trop travailler. Elles fuyaient avec un autre homme de leur choix ou allaient chercher refuge à la mission. L'une des questions majeures est la protection de leurs biens liée à la revendication du lévirat par la famille en cas de décès du mari. Que le mariage fût coutumier ou chrétien, elles trouvaient les arguments susceptibles de le faire casser. Néanmoins, avec l'affermissement du gouvernement colonial qui impliquait la collusion entre l'administration et les chefs, ceux-ci réussirent à leur tour à renforcer, voire à durcir la vision patriarcale de leurs droits matrimoniaux. Les missionnaires changèrent aussi d'attitude, et se mirent à favoriser les hommes de peur qu'ils ne fuient le mariage chrétien qui limitait leurs privilèges.

Au début du $\mathrm{xx}^{\mathrm{e}}$ siècle, la seule échappatoire qui restait aux femmes devenait la ville où il leur restait la possibilité de refuser le mariage aussi bien coutumier que chrétien. Ce n'est que progressivement que, dans la phase suivante, elles récupérèrent une certaine autonomie, à partir du moment où elles purent se dégager à la fois de la pression des chefs et des conventions sociales « victoriennes ».

Les deux papiers suivants analysent de façon privilégiée la jurisprudence du divorce dans les affaires de polygamie qui, en pays d'islam, demeure la règle jusqu'à aujourd'hui.

18 Nathalie Bernard-Maugiron s'interroge sur le droit égyptien qui, depuis le début du $\mathrm{Xx}^{\mathrm{e}}$ siècle, s'est efforcé de rétablir un certain équilibre entre hommes et femmes dans l'accès à la rupture du mariage. En effet, si les modalités juridiques du divorce sont issues des normes de la sharîa, le droit du statut personnel n'est pas resté à l'écart du mouvement de codification initié en Égypte dès le xix siècle. Néanmoins, le droit au divorce reste très inégal par rapport à celui de l'homme qui peut rompre unilatéralement les liens conjugaux par la répudiation, même si celle-ci a aussi eu tendance à être codifiée.

19 Ce qui fait la différence avec la plupart des pays musulmans d'Afrique noire, c'est qu'on dispose de nombre de textes de lois en langue arabe ainsi que de notes explicatives par lesquelles le législateur a tenté de légitimer certaines réformes, parfois audacieuses pour les milieux conservateurs. La première partie de l'article en fait l'analyse. La femme a reçu, dès les années 1920 , le droit de demander le divorce pour préjudice. Mais, en raison de la difficulté de prouver l'existence dudit préjudice, les motifs le plus souvent avancés par l'épouse restent le défaut d'entretien ou l'absence prolongée du mari. C'est seulement en 1979 que la polygamie, à savoir le remariage de l'époux sans le consentement de sa première épouse (ou de ses premières épouses), fut considérée comme préjudiciable, mais à condition que la femme prouve la réalité du dommage matériel ou moral subi (1985). Enfin, en 2000, la femme obtient en principe le droit de demander le divorce sans contrepartie. Les débats parlementaires de l'époque ont mis en exergue le conservatisme de la société et les nombreux préjugés masculins. C'est aussi seulement à cette date que le mari fut tenu d'informer l'épouse de la répudiation qu'il avait prononcée contre elle.

La seconde partie de l'étude est consacrée aux multiples difficultés rencontrées par l'épouse pour faire reconnaître ses droits légaux. Le caractère conservateur des juges peut s'expliquer par le fait que, jusqu'à présent, il n'existe aucune femme juge dans les tribunaux égyptiens ordinaires, ce qui n'a pourtant pas de fondement juridique officiel (mais qui est justifié par la sharîa a), alors qu'il existe en revanche un faible pourcentage d'avocates. Les obstacles sont de trois sortes: religieux, mais aussi sociaux et 
économiques. Le droit à lui seul ne suffit pas à changer les mentalités sociales et culturelles.

21 Marie-José Jolivet nous entraîne, sur les rapports de genre en matière de polygamie, dans une tout autre région, la Guyane. Pourtant, malgré un héritage culturel très différent, la question se pose encore aujourd'hui de façon comparable chez les Ndjuka, l'une des sociétés nées du marronnage, dont beaucoup d'entre eux vivent à SaintLaurent-du-Maroni. Certes, l'influence africaine a marqué les cultures marronnes; néanmoins, dans le cas de la polygamie et des rapports de genre, la société a démontré ses capacités de création et d'ajustements répétés au changement. Toujours est-il que dans ce groupe à dominante matrilinéaire, on assiste, jusqu'à aujourd'hui, au glissement de la "coutume" à la loi. Le mariage relève d'un ensemble coutumier précis, même si la coutume elle-même est une construction assez récente effectuée par une société au démarrage disparate. Il n'y a pas eu de mariage forcé, mais en revanche le mariage était arrangé ; l'initiative de la négociation revenait au matrilignage du futur marié, représenté par un oncle maternel ; l'accord, une fois acquis, l'affaire devient celle des deux familles concernées. Pour obtenir une deuxième ou une troisième épouse, l'homme et son matrilignage devaient accomplir les mêmes démarches que pour la première. Une fois mariée, la femme passait sous la protection de son mari et sa dépendance économique puisqu'il lui était interdit de descendre seule sur la côte, donc de commercialiser ce qu'elle avait elle-même cultivé. C'est à la mort du conjoint que le mariage prend toute sa signification : jamais les hommes ne sont aussi attentionnés que lorsque leurs épouses sont malades, car le veuf doit rester fidèle à son épouse défunte durant une longue période, seuls des rapports sexuels occasionnels étant autorisés avec ses autres épouses. La veuve n'a même pas cette échappatoire de la polygamie. En revanche, si le lévirat n'existe pas en tant que tel, elle peut attendre sa subsistance de la famille du mari.

Ce qui a perturbé cet ordre, c'est la crise économique qui a exigé des femmes la migration du travail, donc une émancipation garantie par la loi française : la question des «papiers " (d'identité) est devenue centrale, car c'est leur obtention qui détermine l'attribution des allocations familiales (versées au père s'il les possède), et c'est en les demandant que la femme devient indépendante. L'article démontre donc attentivement les raisons pour lesquelles cet acte, apparemment simple, est si rarement exécuté. L'entre-deux s'accommode de bien des manières de faire : pour les femmes, seule la maitrise de la langue française peut faire changer les choses.

Dans le dernier article, Meriem Rodary aborde à propos des Marocaines un sujet qui appelle, à son exemple, de plus amples études sur le reste du continent concernant le «droit du travail » féminin : en effet, se détachant de l'histoire domestique et du droit privé, elle montre à quel point celui-ci interfère avec la sphère publique à l'occasion du droit au travail et du droit du travail. Elle met ainsi en garde contre l'opposition classique entre travail domestique "féminin» et économie "masculine». Rien n'interdit en principe aux femmes de travailler. Ce travail n'est ni exceptionnel, ni récent, ni, en soi, dévalorisé par la société. Mais ce à quoi les mœurs et les conventions sociales aboutissent, c'est freiner l'accès direct des femmes aux bénéfices engendrés par leur travail. De ce point de vue, l'auteure récuse un changement ou un progrès résultant du passage de la société ancienne à la «modernisation » occidentale. De tout temps, le droit au travail a été considéré comme normal. Dès la période romaine les femmes étaient investies dans les activités économiques : travail au champ, fileuse, 
tisseuse, nourrice, employée de maison, musicienne, danseuse, prêtresse, cuisinière, ravaudeuse, aide coiffeuse, sage-femme, etc. Le travail est resté une obligation conjugale dans la période précoloniale où le droit musulman était mâtiné d'un droit coutumier similaire, quant au rôle de la force de travail féminine, au nord et au sud du Sahara : ainsi, c'est à la femme ou plus souvent à son père que la dot était payée par le mari. D'où le rôle essentiel des femmes non seulement dans l'agriculture, mais aussi dans l'artisanat textile et dans un certain nombre d'activités marchandes.

C'est au Xxe siècle seulement que le droit au travail des femmes s'est réduit. Naguère, en effet, le seul handicap était la règle incluse dans la sharîa « d'obéissance de l'épouse à son mari». Or, non seulement cette injonction a été reprise au lendemain de l'indépendance dans la Moudawana ou Code de statut personnel, mais elle a explicitement été complétée par le devoir du mari d'entretenir sa femme. Cela n'empêche d'ailleurs pas les femmes de tous les milieux populaires de travailler, mais ce travail, aujourd'hui comme autrefois, a tendance à ne pas être pris en compte en raison de son caractère "informel », c'est-à-dire non comptabilisé. C'est la division sexuelle du travail qui a permis aux hommes de s'approprier le fruit du travail des femmes. Celles-ci ont toujours adopté des stratégies de contournement pour se protéger, y compris la constitution de corporations professionnelles urbaines. Les textes juridiques l'attestent : même si le droit des paysannes à bénéficier de la valeur de leur travail est rarement reconnu, en revanche elles peuvent avoir gain de cause face à l'accaparement par les hommes des bénéfices de leur artisanat.

Que retirer de cet ensemble? Il complète heureusement les acquis dont le contenu est analysé dans le premier article de ce numéro. Celui-ci, on l'a dit en commençant, confirme les avancées remarquables faites dans la connaissance de la place des femmes en droit privé. L'évolution contrastée des droits de la famille, du couple, et sans doute surtout des stratégies et de l'évolution du divorce est désormais très étudiée et ce recueil en fait le point, aussi bien en langue française qu'en langue anglaise. Les processus d'émancipation des femmes, bien que fort inégaux suivant les régions et les cultures, sont partout en marche. Cela incite à une proposition: élargir désormais, comme le font ici certains articles, le champ des recherches à d'autres domaines que celui du droit privé où, si les monographies se multiplient, elles risquent désormais d'apparaître un tantinet répétitives. Il ne faut pas omettre de replacer les femmes dans une perspective résolument genrée, c'est-à-dire examinant leur place non seulement au sein de la famille, mais dans la société sous tous ses aspects : politique, économique, culturel. Ce qui manque aujourd'hui le plus, et qui n'est guère abordé ici, ce sont les travaux sur le premier de ces termes: l'histoire des droits politiques et des droits civiques des femmes, qui ne furent évidemment pas semblables à ceux des hommes ni dans les temps anciens régis par les coutumes, ni dans le droit colonial. Quand, par exemple, les femmes, et lesquelles, eurent le droit de vote? La réponse varie selon les lieux, selon le type de colonisation, selon le mode d'élection (municipal, territorial, voire national après l'indépendance...), selon aussi la réceptivité locale à des normes juridiques plus ou moins comprises ou acceptées. Les modalités en sont encore fort peu étudiées. Une thèse a naguère été consacrée à ces questions ${ }^{5}$. Les sources juridiques offrent la possibilité de combler ces lacunes, il devient urgent de les interroger en ce sens. 


\section{NOTES}

1. Claude Meillassoux, «Essai d'interprétation du phénomène économique dans les sociétés traditionnelles d'auto-subsistance ", Cahiers d'Études africaines, 1960, 4 : 38-67 ; Femmes, greniers et capitaux, Paris, Maspero, 1975.

2. Claude RIVIÈRE, "La promotion de la femme guinéenne ", Cahiers d'Études africaines, 1968, VIII (3), $31:$ 406-427.

3. Lindsay B. SHADLE, « Girl Cases » : Marriage and Colonialism in Gusiiland, Kenya, 1890-1970, Portsmouth, Heinemann, 2006.

4. Margaret KINSMAN, « Beasts of Burden : the Subordination of Southern Tswana Women, ca. 1800-1840 ", Journal of Southern African Studies, 1983, 10 (1) : 39-53.

5. Joséphine BітотA MUAмBA, Recherches sur le statut juridique des femmes en Afrique, thèse de $3^{\text {e }}$ cycle, 2 vol., Université de Toulouse, 2003.

\section{AUTEUR}

\section{CATHERINE COQUERY-VIDROVITCH}

Professeure émérite, Université Paris Diderot-Paris 7/CNRS. 